ESJ Natural/Life/Medical Sciences

\title{
Dynamique Spatiale Et Temporelle De La Sensibilité d'Anopheles Gambiae Sensu Lato Aux Insecticides Utilisés En Santé Publique Au Congo De 2002 À 2018
}

\author{
Grâce Odéra Tainsie Nianga Bikouta, Phd Student \\ Laboratoire de Biodiversité et d'Ecologie Animale (LBDEA), Faculté des \\ Sciences et Techniques \\ Institut National de Recherche en Sciences de la Santé (IRSSA), Ministère \\ de la Recherche Scientifique et de l'Innovation Technologique, Congo \\ Patrick Bitsindou, PhD
}

Point Focal pour la lutte intégrée contre les vecteurs, Ministère de la Santé et de la Population, Congo

\section{Arsène Lenga, $\mathrm{Pr}$}

Laboratoire de Biodiversité et d'Ecologie Animale (LBDEA), Faculté des

Sciences et Techniques, Université Marien Ngouabi, Congo

Doi:10.19044/esj.2021.v17n3p244

Submitted: 29 July 2020

Accepted: 04 January 2020

Published: 31 January 2020
Copyright 2021 Author(s)

Under Creative Commons BY-NC-ND

4.0 OPEN ACCESS

Cite As:

Tainsie Nianga Bikouta G.O., Bitsindou P. \& Lenga A. (2021). Dynamique Spatiale Et Temporelle De La Sensibilité d'Anopheles Gambiae Sensu Lato Aux Insecticides Utilisés En Santé Publique Au Congo De 2002 À 2018. European Scientific Journal, ESJ, 17(3), 244. https://doi.org/10.19044/esj.2021.v17n3p244

\section{Résumé}

En dépit des méthodes de lutte développées contre le paludisme, l'apparition de la résistance d'Anopheles gambiae s.l. aux insecticides constitue une menace susceptible de conduire à l'échec de la lutte contre cette pathologie. Une étude récapitulative a été réalisée afin de montrer la dynamique spatio-temporelle de la résistance d'Anopheles gambiae s.l. à quelques insecticides utilisés au Congo de 2002 à 2018. Les tests de sensibilité ont été réalisés avec des femelles adultes âgées de un à cinq jours. Les insecticides testés appartiennent à 4 familles : (1) organochlorés : DDT 4\%; (2) organophosphorés : malathion 5\%, pirimiphos-méthyl 1,25\% ; (3) pyréthrinoïdes : perméthrine $0,75 \%$, deltaméthrine $0,05 \%$ et $0,5 \%$, 
lambdacyhalothrine $0,05 \%$; (4) carbamates : bendiocarbe $0,1 \%$ et $0,5 \%$. Les tests ont été faits avec les synergistes, l'effet knock-down a été évalué pendant 60 minutes et la mortalité observée 24 heures après.

Cette étude a montré un développement de la résistance au DDT, aux pyréthrinoïdes, l'apparition de la résistance au bendiocarbe et au pirimiphosméthyl et la sensibilité au malathion. Les mutations knockdown resistance (kdr) (L1014F) et (L1014S) sont perçues comme responsables de la résistance au DDT et aux pyréthrinoïdes. Le rétablissement de la sensibilité des anophèles à la deltaméthrine après exposition aux synergistes implique une résistance métabolique.

L'étude a confirmé l'existence de la résistance au DDT, aux pyréthrinoïdes, au bendiocarbe et au pirimiphos-méthyl dans plusieurs départements du pays. Pour garantir l'efficacité des insecticides, il est primordial de surveiller la dynamique de la résistance aux insecticides et éventuellement, identifier les mécanismes impliqués dans la résistance.

Mots-clés: Paludisme, Anopheles Gambiae S.L., Insecticide, Synergiste, Résistance, Congo

\section{Spatial And Temporal Dynamics Of Anopheles Gambiae Sensu Lato Susceptibility To Insecticides Used In Public Health In Congo From 2002 To 2018}

\section{Grâce Odéra Tainsie Nianga Bikouta, Phd Student}

Laboratoire de Biodiversité et d'Ecologie Animale (LBDEA), Faculté des

Sciences et Techniques

Institut National de Recherche en Sciences de la Santé (IRSSA), Ministère

de la Recherche Scientifique et de l'Innovation Technologique, Congo

\section{Patrick Bitsindou, PhD}

Point Focal pour la lutte intégrée contre les vecteurs, Ministère de la Santé et de la Population, Congo

\section{Arsène Lenga, $\mathrm{Pr}$}

Laboratoire de Biodiversité et d'Ecologie Animale (LBDEA), Faculté des

Sciences et Techniques, Université Marien Ngouabi, Congo

\section{Abstract}

Despite control methods developed against malaria, the emergence of Anopheles gambiae s.l. resistance to insecticides constitutes a threat that could lead to the failure of the fight against this pathology. A summary study was carried out in order to show the spatio-temporal dynamic of resistance of 
Anopheles gambiae s.l. to some insecticides used in Congo from 2002 to 2018. Sensitivity tests were carried out with two to five days old adult females. The insecticides tested belonged to 4 families: (1) organochlorines: DDT 4\%; (2) organophosphates: malathion 5\%, pirimiphos-methyl 1.25\%; (3) pyrethroids: permethrin $0.75 \%$, deltamethrin $0.05 \%$ and $0.5 \%$, lambdacyhalothrin $0.05 \%$; (4) carbamates: bendiocarb $0.1 \%$ and $0.5 \%$. Tests were done with synergists. The knock-down effect was evaluated for 60 minutes and mortality 24 hours later. This study showed the existence of resistance to DDT, pyrethroids, development of resistance to bendiocarb and pirimiphos-methyl and sensitivity to malathion in several departments of the country. The knockdown resistance (kdr) mutations (L1014F) and (L1014S) are responsible for DDT resistance. Restoration of Anopheles' sensitivity to deltamethrin after exposure to synergists involves metabolic resistance.

The study confirmed the development susceptibility to DDT, pyrethroids, bendiocarb and pirimiphos-methyl. To ensure the effectiveness of insecticides, it is essential to monitor the periodically susceptibility tests to insecticides and to identify the possible resistance mechanisms involved

Keywords: Malaria, Anopheles Gambiae S.L., Insecticide, Synergists, Resistance, Congo

\section{Introduction}

Le paludisme est une maladie parasitaire potentiellement mortelle. En 2018, l'OMS a estimé l'incidence du paludisme à 228 millions de cas à travers le monde, dont 405000 décès (WHO, 2019).

$\mathrm{Au}$ Congo, le paludisme est stable et sa transmission permanente. Cette infection est le principal facteur de morbidité. En effet, en 2017, il a été enregistré 297652 cas de paludisme dans les structures sanitaires publiques (PNLP, 2018b).

Pour lutter contre cette endémie, la stratégie nationale en matière de lutte contre les vecteurs du paludisme repose essentiellement sur la lutte contre les adultes au moyen de l'utilisation des Moustiquaires Imprégnées d'Insecticide à Longue Durée d'Action (MIILDA). L'utilisation de la MIILDA est devenue l'un des piliers majeurs de la politique de prévention du paludisme au Congo. En effet, son utilisation a été adoptée comme mesure principale de lutte dans le plan stratégique 2008-2012 (PNLP, 2018a).

Cependant, les avantages apportés par l'utilisation de ce moyen de lutte sont menacés par l'émergence de la résistance des anophèles aux insecticides. La résistance d'Anopheles gambiae s.l. aux insecticides est largement répandue en Afrique subsaharienne (Yahouédo et al., 2016, Antonio-Nkondjio et al., 2015 ; Konaté et al., 2016). 
Au Congo, la résistance d'An. gambiae s.l. a été mise en évidence au début des années 2000 essentiellement à Djambala, à Pointe-Noire, à Nkayi, à Owando et à Sibiti (Bitsindou et al., 2006). Depuis lors, ce phénomène s'est étendu dans de nombreuses localités du pays.

Cette situation est d'autant plus inquiétante que l'emploi de MIILDA comme moyens de prévention est fortement adopté par le Programme National de Lutte contre le Paludisme (PNLP) (PNLP, 2018a). Dans un contexte de lutte contre le paludisme et de développement de la résistance des vecteurs aux insecticides, il est nécessaire de suivre régulièrement l'évolution de la sensibilité des vecteurs afin d'initier des mesures de gestion efficaces. L’objectif de la présente étude est de caractériser l'évolution spatio-temporelle et le statut de la résistance des anophèles du complexe gambiae aux insecticides utilisés en santé publique dans différentes localités du Congo de 2002 à 2018. Il s'agit d'une étude intégrant les données récapitulatives sur la sensibilité des vecteurs aux insecticides au Congo de 2002 à 2018 (Itoua et al., 2003; Koekemoer et al., 2011; PNLP, 2010, 2016; Bitsindou, 2014).

\section{Materiels Et Methodes Site d'étude}

Les femelles d’An gambiae s.l. ayant servi à la réalisation des tests mis en œuvre dans cette étude ont été échantillonnées dans 13 localités du pays. Ces localités sont représentatives des principaux faciès éco-épidémiologiques du Congo. Dans le tableau 1 ci-après, sont consignés tous les sites de collecte et les périodes de réalisation des tests.

Tableau 1. Localités de collecte des anophèles et période de réalisation des tests de sensibilité aux insecticides au Congo de 2002 à 2018

\begin{tabular}{|c|c|c|c|c|}
\hline $\begin{array}{c}\text { Départem } \\
\text { ent }\end{array}$ & Localité & Période de collecte & Latitude & $\begin{array}{c}\text { Longitud } \\
\text { e }\end{array}$ \\
\hline Plateaux & Djambala & $\begin{array}{c}\text { septembre 2002-mai 2003, octobre } \\
\text { 2015, avril 2016 }\end{array}$ & $2^{\circ} 32^{\prime} 41^{\prime \prime}$ & $14^{\circ} 45^{\prime} 12^{\prime \prime}$ \\
\hline $\begin{array}{c}\text { Cuvette- } \\
\text { Ouest }\end{array}$ & Ewo & septembre 2002-mai 2003 & $0^{\circ} 52^{\prime} 21^{\prime \prime}$ & $14^{\circ} 49^{\prime} 14^{\prime \prime}$ \\
\hline Likouala & Impfondo & septembre 2002-mai 2003 & $1^{\circ} 37^{\prime} 12^{\prime \prime}$ & $18^{\circ} 03^{\prime} 18^{\prime \prime}$ \\
\hline Bouenza & Nkayi & septembre 2002-mai 2003 & $4^{\circ} 9^{\prime} 56^{\prime \prime}$ & $13^{\circ} 17^{\prime} 34^{\prime \prime}$ \\
\hline $\begin{array}{c}\text { Pointe- } \\
\text { Noire }\end{array}$ & $\begin{array}{c}\text { Pointe- } \\
\text { Noire }\end{array}$ & $\begin{array}{c}\text { septembre 2002-mai 2003, octobre } \\
\text { 2015, avril 2016 }\end{array}$ & $-4^{\circ} 46^{\prime} 33^{\prime \prime}$ & $11^{\circ} 51^{\prime} 48^{\prime \prime}$ \\
\hline Kouilou & Boutoto & avril 2009 & $4^{\circ} 40^{\prime} 31^{\prime \prime}$ & $11^{\circ} 58^{\prime} 14^{\prime \prime}$ \\
\hline $\begin{array}{c}\text { Lékoumou } \\
\text { Cuvette }\end{array}$ & Owandi & $\begin{array}{c}\text { septembre 2002-mai 2003, octobre } \\
2015, \text { avril 2016 }\end{array}$ & $3^{\circ} 44^{\prime} 42^{\prime \prime}$ & $13^{\circ} 24^{\prime} 45^{\prime \prime}$ \\
\hline Sangha & Ouesso & $\begin{array}{c}\text { septembre 2002-mai 2003, octobre } \\
2015, \text { avril 2016 }\end{array}$ & $1^{\circ} 28^{\circ} 66^{\prime \prime} 59^{\prime \prime}$ & $15^{\circ} 54^{\prime} 0^{\prime \prime}$ \\
\hline Pool & Kinkala & Avril 2013 & $4^{\circ} 22^{\prime} 18^{\prime \prime}$ \\
\hline Pool $22^{\prime \prime}$ & Kintélé & octobre17 & $4^{\circ} 9^{\prime} 45^{\prime \prime} 56^{\prime \prime}$ & $15^{\circ} 20^{\prime} 32^{\prime \prime}$ \\
\hline
\end{tabular}




\begin{tabular}{|c|c|c|c|c|} 
Pool & $\begin{array}{c}\text { Djoumou } \\
\text { na }\end{array}$ & septembre 2017-decembre 2018 & $4^{\circ} 22^{\prime} 34^{\prime \prime}$ & $15^{\circ} 9^{\prime} 36^{\prime \prime}$ \\
\hline $\begin{array}{c}\text { Brazzavill } \\
\text { e }\end{array}$ & $\begin{array}{c}\text { Brazzavil } \\
\text { le }\end{array}$ & $\begin{array}{c}\text { mars - avril 2013; avril 2016 - juillet } \\
2018\end{array}$ & - & $15^{\circ} 16^{\prime} 59^{\prime \prime}$ \\
\hline
\end{tabular}

\section{Collecte des moustiques}

Deux méthodes d'échantillonnage ont été utilisées pour obtenir des imagos suivant le protocole de l'OMS (WHO, 1975; OMS, 2014).

La première approche a été celle de la méthode du dipping. Elle a consisté à collecter les larves d'anophèles dans les gîtes larvaires à l'aide de louches. Les larves récoltées ont été transportées au laboratoire dans des bocaux contenant de l'eau des gîtes de prélèvement. Elles ont ensuite été élevées jusqu'à l'émergence des adultes. Les imagos obtenus ont été nourris à l'eau sucrée (sucre de canne). Dans l'insectarium, la température a été maintenue à $27^{\circ} \mathrm{C} \pm 2^{\circ} \mathrm{C}$ avec une humidité relative de $70 \pm 10 \% \mathrm{HR}$.

La seconde approche a été la collecte de la faune résiduelle dans les chambres à coucher. Les femelles gorgées, semi-gravides ou gravides collectées ont été mises en élevage au laboratoire jusqu'à l'obtention des pontes. Les tests de sensibilité ont été réalisés avec des femelles de première génération (F1) à jeun, âgées de 1 à 5 jours issues de ces pontes.

Les anophèles femelles utilisés dans les tests ont été identifiés morphologiquement comme appartenant au complexe Anopheles gambiae en utilisant les clés de Gillies et De Meillon (1968) (Gillies and De Meillon, 1968) et de Gillies et Coetzee (1987) (Gillies and Coetzee, 1987).

\section{Insecticides testés}

Les papiers imprégnés d'insecticide ont été obtenus par l'intermédiaire de l'OMS/AFRO auprès de son centre de référence (Unité de recherche sur le contrôle des vecteurs, Université Sains Malaysia, Penang, Malaisie).

Six insecticides (sur papiers imprégnés) appartenant à quatre familles ont été testés aux concentrations suivantes :

- $\quad$ Organochlorés DDT 4\%;

- $\quad$ Organophosphorés : malathion 5\% et pirimiphos-méthyl 1,25\%;

- $\quad$ Carbamates : bendiocarbe $0,01 \%$ et $0,5 \%$;

- Pyréthrinoïdes : perméthrine $0,75 \%$; deltaméthrine $0,05 \%$ et $0,5 \%$ et lambdacyhalothrine $0,05 \%$.

\section{Essais biologiques de sensibilité aux insecticides}

Les tests de sensibilité ont été effectués sur des femelles d'An. gambiae s.l. à jeun âgées de 1 à 5 jours, selon le protocole standard OMS pour adultes à l'aide de kits OMS (WHO 1998, OMS 2017). Les tests ont été réalisés sous une température ambiante et une humidité relative respectivement de $25 \pm 2^{\circ} \mathrm{C}$ et $70 \pm 10 \%$ HR. Pour chaque test, neuf tubes ont été utilisés. Dans chaque tube, 
les femelles ont été introduites par lot d'environ 25 femelles. Chaque test a été réalisé en deux temps: dans un premier temps, les lots de moustiques ont été mis en observation pendant une heure dans des tubes pourvus de papier nonimprégné (12 x $15 \mathrm{~cm}$ ); dans un deuxième temps, les femelles ont été mises en contact avec l'insecticide, dans des tubes pourvus de papier imprégné (12 x $15 \mathrm{~cm}$ ), tandis que les femelles servant de témoins ont été introduites dans des tubes pourvus de papier non-imprégné.

Au cours de la période d'exposition de 60 minutes, l'effet Knock-down a été observé toutes les 5 minutes. À la fin de cette période, les femelles ont été transférées dans des tubes d'observation. Un tampon de coton imbibé d'une solution sucrée (sucre de canne) à 10\% a été alors placé sur chaque tube d'observation pour l'alimentation des moustiques permettant ainsi d'écarter la faim comme cause de mortalité. La mortalité est ensuite évaluée 24 heures après l'exposition à l'insecticide (WHO, 1998; OMS, 2017). La souche sensible SUA (colonie susceptible) n'a été utilisée que pour l'étude de Boutoto en 2009. Pour les autres études, aucune souche de référence sensible n’a été utilisée. L’absence de la souche Kisumu habituellement utilisée pour évaluer ou confirmer, lors des tests de sensibilité, la rémanence d'un insecticide constitue une limite quant à la validité de nos tests. Cependant, étant donné (i) que les papiers imprégnés utilisés proviennent du centre de référence (Unité de recherche sur le contrôle des vecteurs, Université Sains Malaysia, Penang, Malaisie) ; (ii) que ces papiers ont été utilisés pendant leur période de validité et (iii) la répétition des tests dans les mêmes conditions associée à l'absence de mortalité chez les témoins... ces critères garantissent la présence de l'insecticide et la qualité de l'imprégnation des papiers utilisés et confortent les résultats obtenus au cours des différents tests.

\section{Bio-essais synergiques}

Afin d'évaluer l'implication des enzymes detoxifiantes dans la résistance à la deltaméthrine (insecticide utilisé dans l'imprégnation de moustiquaires au Congo) et au DDT, des tests avec des synergistes ont été réalisés avec une préexposition au $\mathrm{PBO} 4 \%$ (butoxyde de pipéronyle) inhibiteur principalement des mono-oxygénases; au maléate de diéthyle 20\% inhibiteur de glutathion-S-transférases (GST) et au TPP 20\% (triphényle phosphate) un inhibiteur des estérases (Brogdon et Barber, 1990; William et Janet, 1997; Chareonviriyaphap et al., 2003; OMS, 2017). Chaque test a été réalisé en trois temps avec quatre lots constitués de 20 à 25 femelles d'An. gambiae s.l. non gorgées âgés de 1 à 5 jours. Les quatre lots ont été répartis comme suit: 1 lot à tester au synergiste seul, 1 lot à tester à l'insecticide (deltaméthrine $0.05 \%$ ou DDT $4 \%$ ) seul, 1 lot à tester au synergiste puis à l'insecticide et 1 lot servant de témoin exposé à du papier non-imprégné. Dans un premier temps, les lots de femelles ont été mis en observation pendant une 
heure dans des tubes pourvus de papier non-imprégné $(12$ x $15 \mathrm{~cm})$. Dans un deuxième temps, 2 lots de 20 à 25 femelles ont été introduits chacun dans un tube tapissé de papier imprégné de synergiste pendant 1 heure. Enfin dans un troisième temps, les femelles testées au synergiste seul ont été retransferés dans le tube d'observation, et dans les trois autres lots, les femelles ont été transférées dans les tubes d'exposition. Le transfert a été fait de la manière suivante : le lot à tester au synergiste puis à l'insecticide a été transféré dans le tube contenant de l'insecticide, le lot à tester à l'insecticide a été transféré dans 1 tube contenant un papier imprégné d'insecticide et le lot témoin a été transféré dans 1 tube contenant du papier non imprégné. Après 1 heure d'exposition, les trois lots ont été retransferés dans leurs tubes d'observation respectifs fournis avec une solution d'eau sucrée (sucre de canne) à 10\% et maintenue pendant 24 heures avant l'enregistrement de la mortalité.

\section{Critère de validité des tests de sensibilité}

La sensibilité des populations a été classée selon les critères de l'OMS et les tests de sensibilité ont été réalisés, selon les critères OMS (WHO 1998; OMS 2017). L'effet knock-down de l'insecticide a été évalué pendant la période d'exposition qui a duré 1 heure et son effet létal a été évalué 24 heures après la période d'exposition. Les critères d'interprétation utilisés sont consignés dans le tableau 2 ci-dessous.

Tableau 2 : Présentation des critères d'interprétation des tests de sensibilité.

\begin{tabular}{|c|c|}
\hline Taux de mortalité & Interprétation \\
\hline compris entre $98-100 \%$ & population sensible à l'insecticide testé \\
\hline compris entre $90-97 \%$ & baisse de sensibilité au sein de la population \\
\hline inférieur à $90 \%$ & population résistante à l'insecticide testé \\
\hline
\end{tabular}

Pour les tests avec les synergistes, les critères de validité ont été appliqués selon les recommandations des travaux précédents (William et Janet, 1997; Brogdon et Barber, 1990; Chareonviriyaphap et al., 2003; OMS, 2017).

Pour la validation des résultats, la mortalité des témoins doit être inférieure à 5\%. Lorsqu'elle est comprise entre 5\% et 20\%, la formule d'Abbot est utilisée pour corriger la mortalité des moustiques exposés. Mais lorsqu'elle est supérieure à $20 \%$, le test est annulé (Abbott, 1925). Cette formule n’a été utilisée que pour les tests faits entre 2002 et 2003 du fait que la mortalité chez les témoins était de $8 \%$.

\section{Traitement statistique des données}

Au cours de cette étude, les données ont été saisies avec le logiciel Excel 2010 et traitées avec les tests de statistique de Fisher exact et/ou Pearson 
Chi2 pour comparer les taux de mortalité. Le test de Student a été utilisé pour comparer les taux de mortalité des tests avec les synergistes.

Une valeur de probabilité $\mathrm{p}$ inférieure ou égale à 0,05 a été considérée comme significative. Les analyses statistiques ont été effectuées à l'aide du logiciel Xlstat (version 2019.3.2).

\section{Resultats}

Les taux de mortalité induits par les insecticides testés sur An. gambiae s.l. montrent que la résistance aux insecticides est un processus dynamique qui se traduit par un nombre élevé d'individus survivants aux tests de sensibilité. Les détails des résultats sont consignés, selon chaque insecticide, dans les tableaux 3 à 10 et les figures 1 et 2.

Tableau 3. Mortalité d'An. gambiae s.l. au DDT 4\% et à dieldrine 4\% dans 12 localités du Congo entre 2002-2018.

\begin{tabular}{|c|c|c|c|}
\hline Localités & Date & \% mortalité $(\mathrm{N})$ & Statut \\
\hline \multicolumn{4}{|c|}{ DDT 4\% } \\
\hline \multirow{2}{*}{ Brazzaville } & $2013-2014$ & $2 \%(100)$ & $\mathrm{R}$ \\
\hline & 2016-2018 & $8 \%(100)$ & $\mathrm{R}$ \\
\hline \multirow{2}{*}{ Djambala } & $2002-2003$ & $90 \%(80)$ & BS \\
\hline & 2015-2016 & $7 \%(88)$ & $\mathrm{R}$ \\
\hline \multirow{2}{*}{ Ewo } & $2002-2003$ & $100 \%(80)$ & $\mathrm{S}$ \\
\hline & 2012-2013 & $100 \%(100)$ & $\mathrm{S}$ \\
\hline Impfondo & $2002-2003$ & $92 \%(95)$ & BS \\
\hline Kinkala & 2013-2014 & $88 \%(100)$ & $\mathrm{R}$ \\
\hline Nkayi & $2002-2003$ & $92,4 \% *(100)$ & $\mathrm{BS}$ \\
\hline \multirow{2}{*}{ Ouesso } & 2010 & $4 \%(88)$ & $\mathrm{R}$ \\
\hline & 2015-2016 & $4 \%(100)$ & $\mathrm{R}$ \\
\hline Owando & 2013-2014 & $89 \%(100)$ & $\mathrm{R}$ \\
\hline \multirow{2}{*}{ Pointe-Noire } & $2002-2003$ & $17 \%(88)$ & $\mathrm{R}$ \\
\hline & 2010 & $33 \%(84)$ & $\mathrm{R}$ \\
\hline Sibiti & $2002-2003$ & $96 \%(95)$ & BS \\
\hline Djoumouna & 2016-2018 & $6 \%(100)$ & $\mathrm{R}$ \\
\hline Boutoto & 2009 & $0 \%(64)$ & $\mathrm{R}$ \\
\hline
\end{tabular}

Statut : R = résistant, $\mathbf{S}$ = sensible, $\mathbf{B S}$ = baisse de sensibilité ; *=mortalité corrigée;

$$
\mathbf{N}=\text { effectif testé }
$$

Les tests réalisés entre 2002 et 2018 indiquent que les souches d’An. gambiae s.l. sont résistantes au DDT et à la dieldrine et en baisse de résistance dans toutes les localités. A l'exception d'Ewo où les populations testées ont été sensibles au DDT.

Tableau 4 : Mortalité d’An. gambiae s.l. à la perméthrine 0,75\% dans 9 localités du Congo entre 2002-2018.

\begin{tabular}{|c|ccc|}
\hline Localités & Date & \% mortalité (N) & Statut \\
\hline & Perméthrine $0,75 \%$ & \\
\hline \multirow{2}{*}{ Brazzaville } & $2013-2014$ & $46 \%(100)$ & $\mathrm{R}$ \\
& $2016-2018$ & $43,4 \%(100)$ & $\mathrm{R}$ \\
\hline Djambala & $2002-2003$ & $97,8 \% *(80)$ & BS
\end{tabular}




\begin{tabular}{|c|ccc|} 
& $2015-2016$ & $53 \%(84)$ & $\mathrm{R}$ \\
\hline Ewo & $2002-2003$ & $100 \%(80)$ & $\mathrm{S}$ \\
\hline Impfondo & $2002-2003$ & $100 \%(91)$ & $\mathrm{S}$ \\
\hline Nkayi & $2002-2003$ & $89 \% *(106)$ & $\mathrm{R}$ \\
\hline Ouesso & 2010 & $20 \%(96)$ & $\mathrm{R}$ \\
\hline Owando & $2002-2003$ & $66 \%(100)$ & $\mathrm{R}$ \\
\hline \multirow{3}{*}{ Pointe-Noire } & $2002-2003$ & $95 \%(80)$ & $\mathrm{BS}$ \\
& 2010 & $91 \%(84)$ & $\mathrm{BS}$ \\
& $2015-2016$ & $75 \%(100)$ & $\mathrm{R}$ \\
\hline \multirow{2}{*}{ Sibiti } & $2002-2003$ & $99 \% *(80)$ & $\mathrm{S}$ \\
& $2015-2016$ & $76 \%(100)$ & $\mathrm{R}$ \\
\hline
\end{tabular}

Statut : R = résistant, $\mathbf{S}$ = sensible, $\mathbf{B S}$ = baisse de sensibilité; *= mortalité corrigée; $\mathbf{N}=$ effectif testé

Les tests récents montrent l'existence de la résistance des souches d'An. gambiae s.l. notamment à Sibiti, à Pointe-Noire, à Djambala et à Brazzaville.

Tableau 5. Mortalité d'An. gambiae s.l. à la deltaméthrine 0,05\% et 0,5\% dans 11 localités du Congo entre 2002-2018.

\begin{tabular}{|c|c|c|c|}
\hline Localités & Date & \% mortalité $(\mathrm{N})$ & Statut \\
\hline \multicolumn{4}{|c|}{ Deltaméthrine $0,05 \%$} \\
\hline \multirow{2}{*}{ Brazzaville } & 2013-2014 & $89 \%(100)$ & $\mathrm{R}$ \\
\hline & $2016-2018$ & $73,1 \%(100)$ & $\mathrm{R}$ \\
\hline Boutoto & 2009 & $74 \%(100)$ & $\mathrm{R}$ \\
\hline \multirow{2}{*}{ Djambala } & $2002-2003$ & $76 \%(80)$ & $\mathrm{R}$ \\
\hline & $2015-2016$ & $56 \%(88)$ & $\mathrm{R}$ \\
\hline Djoumouna & 2016-2018 & $82 \%(100)$ & $\mathrm{R}$ \\
\hline Impfondo & $2002-2003$ & $100 \%(100)$ & $\mathrm{S}$ \\
\hline Nkayi & $2002-2003$ & $98 \% *(100)$ & S \\
\hline \multirow{2}{*}{ Ouesso } & 2010 & $79 \%(88)$ & $\mathrm{R}$ \\
\hline & $2015-2016$ & $81 \%(100)$ & $\mathrm{R}$ \\
\hline Owando & $2002-2003$ & $100 \% *(100)$ & $\mathrm{S}$ \\
\hline \multirow{3}{*}{ Pointe-Noire } & $2002-2003$ & $98,9 \%(100)$ & $\mathrm{S}$ \\
\hline & 2010 & $89 \%(88)$ & $\mathrm{R}$ \\
\hline & $2015-2016$ & $48 \%(100)$ & $\mathrm{R}$ \\
\hline \multirow{2}{*}{ Sibiti } & $2002-2003$ & $88,9 \%(80)$ & $\mathrm{R}$ \\
\hline & $2015-2016$ & $84 \%(100)$ & $\mathrm{R}$ \\
\hline \multicolumn{4}{|c|}{ Deltaméthrine 0,5\% } \\
\hline Brazzaville & 2018 & $89,75 \%(100)$ & $\mathrm{R}$ \\
\hline Djoumouna & 2018 & $100 \%(100)$ & $\mathrm{S}$ \\
\hline Kintélé & 2017 & $100 \%(75)$ & $\mathrm{S}$ \\
\hline
\end{tabular}

Statut : $\mathbf{R}$ = résistant, $\mathbf{S}$ = sensible; *=mortalité corrigée; $\mathbf{N}=$ =effectif testé

Comme pour la perméthrine, les résultats des tests récents montrent également une résistance des souches d'An. gambiae s.l. à la deltaméthrine notamment à Pointe-Noire, à Sibiti, à Djambala et à Brazzaville. Les résultats 
des tests montrent en outre que les souches testées sont sensibles à la deltaméthrine $0,5 \%$; seules les souches de Brazzaville collectées dans différents quartiers de la ville ont présenté une résistance à la deltaméthrine.

Tableau 6. Mortalité d'An. gambiae s.l. à la lambdacyhalothrine 0,05\% dans 10 localités du Congo entre 2002-2018.

\begin{tabular}{|c|ccc|}
\hline Localité & Date & $\%$ mortalité (N) & Statut \\
\hline & $2013-2014$ & $85 \%(100)$ & $\mathrm{R}$ \\
\hline \multirow{2}{*}{ Brazzaville } & $2016-2018$ & $49 \%(100)$ & $\mathrm{R}$ \\
\hline \multirow{2}{*}{ Djambala } & $2002-2003$ & $85 \%(80)$ & $\mathrm{R}$ \\
& $2015-2016$ & $68 \%(88)$ & $\mathrm{R}$ \\
\hline Djoumouna & $2016-2018$ & $29 \%(100)$ & $\mathrm{R}$ \\
\hline Ewo & $2002-2003$ & $100 \%(80)$ & $\mathrm{S}$ \\
\hline Impfondo & $2002-2003$ & $100 \%(90)$ & $\mathrm{S}$ \\
\hline Nkayi & $2002-2003$ & $99 \%(95)$ & $\mathrm{S}$ \\
\hline Ouesso & $2015-2016$ & $57 \%(100)$ & $\mathrm{R}$ \\
\hline Owando & $2002-2003$ & $100 \%(100)$ & $\mathrm{S}$ \\
\hline \multirow{2}{*}{ Pointe-Noire } & $2002-2003$ & $95,8 \%(96)$ & $\mathrm{BS}$ \\
\hline Sibiti & $2015-2016$ & $54 \%(100)$ & $\mathrm{R}$ \\
\hline Nt & $2002-2003$ & $63 \%(80)$ & $\mathrm{R}$ \\
\hline
\end{tabular}

Statut : $\mathbf{R}$ = résistant, $\mathbf{S}$ = sensible, $\mathbf{B S}$ = baisse de sensibilité; $\mathbf{N}=$ effectif testé

Les résultats des tests récents montrent une résistance des souches d'An. gambiae s.l. à la lambdacyhalothrine dans différentes localités mentionnées dans le tableau 6.

Tableau 7. Mortalité d'An. gambiae s.l. au malathion 5\% et au pirimiphos-méthyl 1,25\% dans 6 localités du Congo entre 2013-2018.

\begin{tabular}{|c|ccc|}
\hline Localité & Date & $\%$ mortalité $(\mathrm{N})$ & Statut \\
\hline \multicolumn{5}{|c|}{ Malathion 5\% } \\
\hline Boutoto & 2009 & $100 \%(100)$ & $\mathrm{S}$ \\
\hline Brazzaville & $2013-2014$ & $100 \%(100)$ & $\mathrm{S}$ \\
\hline Ewo & $2013-2014$ & $100 \%(100)$ & $\mathrm{S}$ \\
\hline Kinkala & $2013-2014$ & $100 \%(100)$ & $\mathrm{S}$ \\
\hline Owando & $2013-2014$ & $100 \%(100)$ & $\mathrm{S}$ \\
\hline Djoumouna & $2016-2016$ & $100 \%(100)$ & $\mathrm{S}$ \\
\hline \multicolumn{5}{c}{ Pirimiphos-méthyl 1,25\% } & $\mathrm{R}$ \\
\hline Djoumouna & 2018 & $32 \%(100)$ & \\
\hline
\end{tabular}

\%mort: \% de mortalité ; Statut : $\mathrm{R}$ = résistant, $\mathrm{S}$ = sensible, $\mathrm{BS}$ = baisse de sensibilité; $\mathrm{N}=$ effectif testé

Toutes les souches testées au malathion ont été sensibles à cet insecticide. Par ailleurs, la souche de Djoumouna a été résistante au pirimiphos-méthyl 1,25\% (mortalité 32\%). 
Tableau 8. Mortalité d’An. gambiae s.l. au bendiocarbe 0,1\% dans 6 localités du Congo entre 2009-2018.

\begin{tabular}{|c|c|c|c|}
\hline Localité & Date & $\begin{array}{c}\text { \% mortalité } \\
(\mathrm{N})\end{array}$ & Statut \\
\hline \multicolumn{4}{|c|}{ Bendiocarbe $0,1 \%$} \\
\hline Boutoto & 2009 & $96 \%(106)$ & BS \\
\hline \multirow{2}{*}{ Brazzaville } & $2013-2014$ & $100 \%(100)$ & $\mathrm{S}$ \\
\hline & $2016-2018$ & $84 \%(100)$ & $\mathrm{R}$ \\
\hline Ewo & $2013-2014$ & $100 \%(100)$ & $\mathrm{S}$ \\
\hline Kinkala & $2013-2014$ & $100 \%(100)$ & $\mathrm{S}$ \\
\hline Owando & $2013-2014$ & $100 \%(100)$ & $\mathrm{S}$ \\
\hline Djoumouna & $2016-2018$ & $86 \%(100)$ & $\mathrm{R}$ \\
\hline
\end{tabular}

Statut : $\mathrm{R}$ = résistant, $\mathrm{S}$ = sensible, $\mathrm{BS}$ = baisse de sensibilité; $\mathrm{N}=$ effectif testé

Dans l'ensemble des localités mentionnées, les souches d'An. gambiae s.l. testées ont été sensibles au malathion (organophosphoré).

Pour le bendiocarbe (carbamate), les souches d'An. gambiae s.l. testées à Boutoto en 2009 ont montré une baisse de sensibilité (mortalité de 96\%). En 2013-2014, les populations testées dans les localités mentionnées ont présenté une sensibilité à cet insecticide. Cependant, en 2016-2018, les souches d'An. gambiae s.l. de Brazzaville et de Djoumouna ont présenté une résistance à ce produit, avec des taux de mortalité respectifs de $84 \%$ et de $86 \%$.

Tableau 9. Répartition des taux de mortalité d'An. gambiae s.l. exposé à la deltaméthrine 0,05\% seul versus exposé au préalable au PBO 4\% puis à la deltaméthrine 0,05\% souche de Boutoto en 2009 et de Brazzaville en 2018.

\begin{tabular}{|c|c|c|c|c|}
\hline Insecticide et concentration & \multicolumn{3}{|c|}{ PBO 4\% + deltaméthrine 0,05\% } \\
\hline \multirow{2}{*}{ Localité et année } & $\begin{array}{c}\text { deltaméthrine 0,05\% } \\
\text { seule }\end{array}$ & \multicolumn{2}{c|}{ PBO 4\% + deltaméthrine 0,05\% } \\
\cline { 2 - 5 } & $\begin{array}{c}\text { \% mortalité } \\
(\mathrm{N})\end{array}$ & statut & $\begin{array}{c}\% \\
\text { mortalité } \\
(\mathrm{N})\end{array}$ & statut \\
\hline Boutoto 2009 & $53,7 \%(94)$ & $\mathrm{R}$ & $92,4 \%(91)$ & $\mathrm{BS}$ \\
\hline Brazzaville 2018 & $78,6 \%(75)$ & $\mathrm{R}$ & $98,6 \%(75)$ & $\mathrm{S}$ \\
\hline
\end{tabular}

PBO : butoxyde de pipéronyle; Statut : $\mathbf{R}$ = résistant, $\mathbf{S}=$ sensible, $\mathbf{B S}=$ baisse de sensibilité; $\mathbf{N}=$ effectif testé

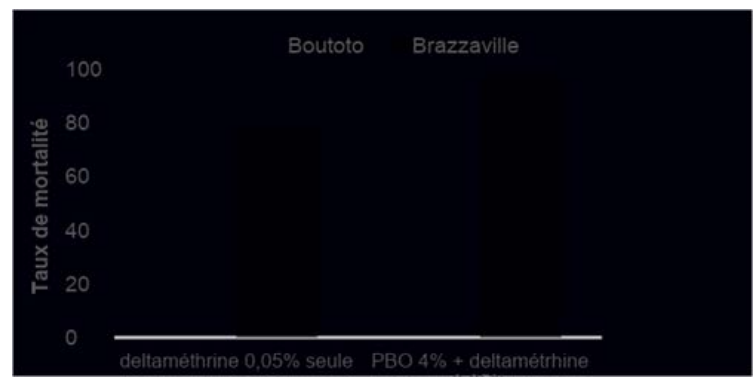


Figure 1. Taux de mortalité d'An. gambiae s.l. exposé à la deltaméthrine 0,05\% seul versus exposé au préalable au $\mathrm{PBO} 4 \%$ puis à la deltaméthrine 0,05\% à Boutoto en 2009 et à Brazzaville en 2018.

Le tableau 9 et la figure 1 montrent les taux de mortalité à la deltaméthrine seule et au PBO + deltaméthrine $0,05 \%$; ceux-ci ont été respectivement de $53,7 \%$ et $92,4 \%$ à Boutoto, de $78,6 \%$ et $98,6 \%$ à Brazzaville. L'exposition au PBO a entrainé une augmentation significative des taux de mortalité dans ces localités. L'analyse de l'impact du PBO sur les taux de mortalité est du même ordre dans les deux localités $(\mathrm{p}<0,149)$.

Tableau 10. Répartition des taux de mortalité d'An. gambiae s.l. exposé à la deltaméthrine 0,05\%, seul versus exposé au préalable au TPP 20\% et diéthylmaleate $20 \%$ puis à la deltaméthrine $0,05 \%$ à Boutoto en 2009.

\begin{tabular}{|c|c|c|c|c|c|c|c|c|}
\hline $\begin{array}{l}\text { Insecticide } \\
\text { et } \\
\text { concentrati }\end{array}$ & \multicolumn{4}{|c|}{ TPP 20\% + deltaméthrine 0,05\% } & \multicolumn{4}{|c|}{$\begin{array}{c}\text { Diéthylmaleate } 20 \%+\text { deltaméthrine } \\
0,05 \%\end{array}$} \\
\hline \multirow[b]{2}{*}{ Localité } & $\begin{array}{r}\text { Deltam } \\
0,05 \%\end{array}$ & $\begin{array}{l}\text { hrine } \\
\text { eule }\end{array}$ & $\begin{array}{r}\text { TPP 20\% } \\
\text { deltaméthr } \\
0,05 \%\end{array}$ & & $\begin{array}{l}\text { Deltamé } \\
0,05 \%\end{array}$ & & $\begin{array}{r}\text { Diéthyl } \\
20 \% \\
\text { deltam } \\
0,0\end{array}$ & $\begin{array}{l}\text { leate } \\
\text { Irine } \\
6\end{array}$ \\
\hline & $\begin{array}{c}\% \\
\text { mortalit } \\
\text { é }(\mathrm{N})\end{array}$ & statut & $\begin{array}{l}\text { \% mortalité } \\
(\mathrm{N})\end{array}$ & $\begin{array}{c}\text { stat } \\
\text { ut }\end{array}$ & $\begin{array}{c}\% \\
\text { mortalit } \\
\text { é }(\mathrm{N})\end{array}$ & $\begin{array}{c}\text { stat } \\
\text { ut }\end{array}$ & $\begin{array}{c}\% \\
\text { mortalité } \\
\text { (N) }\end{array}$ & statut \\
\hline Boutoto & $\begin{array}{c}56,6 \% \\
(99)\end{array}$ & $\mathrm{R}$ & $81,5 \%(101)$ & $\mathrm{R}$ & $\begin{array}{c}43,9 \% \\
(94)\end{array}$ & $\mathrm{R}$ & $\begin{array}{c}43,6 \% \\
(91)\end{array}$ & $\mathrm{R}$ \\
\hline
\end{tabular}

TPP : triphényle phosphate ; Statut : $\mathbf{R}=$ résistant, $\mathbf{S}=$ sensible ; $\mathbf{N}=$ effectif testé

TPP : Triphényle phosphate ; delt : deltaméthrine

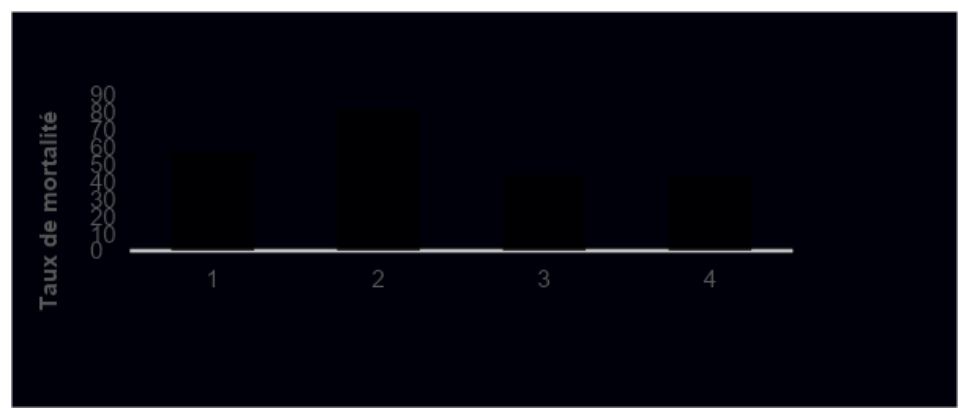

Figure 2. Taux de mortalité d'An. gambiae s.l. exposés à la deltaméthrine 0,05\% avec et sans synergistes (au TPP 20\% et au diethylmaleate 20\%) à Boutoto en 2009.

Le tableau 10 et la figure 2 montrent les taux de mortalité à la deltaméthrine seule au TPP $20 \%$ + deltaméthrine $0,05 \%$; ces taux ont été respectivement de $56,6 \%$ et $81,5 \%$. Les taux de mortalité à la deltaméthrine seule au diéthylmaleate $20 \%$ + deltaméthrine $0,05 \%$ sont demeurés pratiquement inchangés. 


\section{Discussion}

Les résultats présentés dans cette étude montrent des niveaux de résistance inquiétants sur l'évolution de celle-ci dans les localités concernées. Par ailleurs, les tests de sensibilité n’ont pas été faits régulièrement dans toutes les localités entre 2002 et 2018 alors qu'ils auraient dû l'être. Ainsi, il n'a pas été possible de dire avec exactitude quel a été le facteur déterminant dans l'apparition de la résistance dans chaque localité. Cependant, à partir des résultats obtenus, l'évolution spatio-temporelle de la résistance aux insecticides au Congo peut être évaluée. Ainsi, la résistance serait due en partie à l'emploi à grande échelle des MIILDA par les populations. En effet, entre 2011 et 2012, deux millions quatre cent quatre-vingt-un mille cinq cent soixante-trois (2 481563 ) moustiquaires imprégnées de deltaméthrine ont été distribuées à la population au niveau national (PNLP, 2012). L'emploi massif des MIILDA a pu avoir exercé une pression de sélection qui a induit la résistance aux insecticides appartenant à la famille des pyréthrinoïdes. Des études réalisées dans d'autres pays ont montré que l'emploi massif de MIILDA a été un facteur déterminant dans l'apparition de la résistance des anophèles aux pyréthrinoïdes (Yahouédo et al 2016; Thiaw et al 2018). Cette résistance a pu être également confortée par l'utilisation des insecticides de la même famille (tétraméthrine, perméthrine, lambdacyhalothrine) en agriculture. La lutte contre les ravageurs de culture se fait aussi avec d'autres insecticides comme le carbofuran (carbamate nématicide), le diméthoate (organophosphoré utilisé comme insecticide et acaricide), le diazinon (insecticide organophosphoré); l'endosulfan (un insecticide organochloré dont l'utilisation est restreinte ou interdite aux États-Unis et en Europe en raison de sa persistance dans l'environnement, dans l'organisme humain et de sa toxicité modérée) (Kucuker et al., 2009). La résistance à ces divers insecticides pourrait être causée par la résistance métabolique impliquant les enzymes de détoxication, les monooxygénases à cytochrome P450, les glutathion Stransférases (GSTs) et les carboxylestérases (COEs). Ces enzymes sont en effet impliquées dans la résistance aux pyréthrinoïdes contribuant de manière incontestable à la résistance aux insecticides de cette famille (CNEV, 2014). Les insecticides utilisés dans la lutte contre les ravageurs pourraient contaminer les sites d'oviposition des anophèles, ce qui exercerait une pression de sélection importante et constante sur les populations larvaires d'anophèles. Un tel effet peut expliquer à plus ou moins long terme, l'émergence d'une résistance à ces insecticides dans les populations des vecteurs du paludisme.

Ces résultats sont en accord avec ceux obtenus par d'autres auteurs (Alou et al., 2010; Konan et al,, 2011; Abuelmaali et al., 2013; Cisse et al., 2015; Antonio-Nkondjio et al., 2016), qui ont montré que l'utilisation fréquente des insecticides dans les exploitations agricoles et dans le stockage 
de produits agricoles était à l'origine de forts taux de résistance chez $A n$. gambiae s.l. à tous les insecticides testés.

En outre, dans le contexte de cette pression sélective, il est important de signaler également que les évolutions démographique et urbaine ont occasionné l'emploi des aérosols dans les ménages et les pulvérisations de divers insecticides par les compagnies privées opérant dans la lutte contre la nuisance culicidienne. Toutes ces pratiques ont contribué significativement à l'émergence, le maintien et le développement de la résistance chez les vecteurs du paludisme. Au-delà des causes évoquées, il convient de mentionner la présence à Brazzaville, à Pointe-Noire et dans d'autres villes du pays, des industries émettant des substances polluantes et toxiques qui pourraient contribuer aussi à l'apparition de la résistance aux insecticides chez les culicidés.

Ainsi, le maintien des différentes pressions exercées par les insecticides et la transmission probable des gènes de résistance aux descendants ont au fil du temps, favorisé la prolifération d'individus résistants au sein des populations anophéliennes. Ces hypothèses ont été confirmées dans d'autres pays (Chandre et al., 1999; Antonio-Nkondjio et al., 2011; Ndo et al., 2011). Les populations sensibles ou en baisse de sensibilité en 20022003 (Itoua et al., 2003) se sont révélées résistantes aux mêmes insecticides (deltaméthrine, perméthrine et lambdacyhalothrine) en 2015-2016 (PNLP, 2016). Ce qui montre une évolution du phénomène de résistance au sein des populations d'An. gambiae s.l. dans les localités étudiées (Djambala, Sibiti, Pointe-Noire). Cependant le statut de résistance doit être réévalué dans les localités où les tests réalisés en 2002-2003 ont montré une sensibilité du vecteur à ces produits. Le statut général actuel de la résistance du vecteur nécessite la réalisation des tests dans l'ensemble des localités de manière régulière, au moins tous les deux ans.

En 2013, à Brazzaville, toutes les populations testées provenaient d'un mélange de larves issues de différents quartiers de la ville. Ces populations présentaient une résistance au DDT et aux pyréthrinoïdes (deltaméthrine, perméthrine et lambdacyhalothrine) (Bitsindou, 2014).Comme cela était également observé dans d'autres pays africains (Akogbéto et Yakoubou, 1999; Antonio-Nkondjio et al., 2011; Jones et al., 2012).

Les tests de 2016-2018 ont été faits avec des populations collectées dans différents quartiers (Makélékélé, jardin d'essai, Bacongo, Moungali, Kombo et Mayanga), il faut noter que, seules les populations d'An. gambiae s.l. des quartiers Bacongo et Mayanga ont été sensibles à la deltaméthrine. Ces résultats ont montré que la résistance à une famille d'insecticides varie d'un quartier à un autre, probablement en fonction des activités anthropiques qui y sont menées. En 2018, les tests faits dans les mêmes quartiers ont montré que les populations testées étaient devenues résistantes à la deltaméthrine 
traduisant une perte progressive de la sensibilité d'An. gambiae s.l. dans ces quartiers et le maintien de la résistance dans les autres. En effet, dans le quartier Mayanga les collectes ont été faites dans un site de maraîchage, donc un milieu potentiellement soumis aux insecticides agricoles. La perte de la sensibilité à la deltaméthrine et la résistance à la perméthrine et au DDT seraient due à une intensification de l'emploi des insecticides comme cela a été constaté sur le terrain. Des insecticides tels que la tétraméthrine, la perméthrine, la lambdacyhalothrine le carbofuran, le diméthoate, le diazinon et endosulfan y sont utilisés. De ce fait, ces anophèles soumis à une pression insecticide constante résistent aux pyréthrinoïdes et au DDT en modifiant le site cible d'action de l'insecte ou avec les enzymes detoxifiantes entraînant la dégradation des pyréthrinoïdes, du DDT et aussi des insecticides appartenant aux familles des carbamates et des organophosphorés en métabolites moins toxiques ou inactifs et plus facilement excrétables (CNEV, 2014).

Même si le DDT n'est plus homologué au Congo (MEFDDE, 2017), ce produit a été largement utilisé durant la période coloniale et celle qui a suivi l'indépendance (Adam, 1964). La résistance des vecteurs au DDT pourrait être liée à cette pratique et être amplifiée par l'utilisation à large échelle au Congo des MIILDA imprégnées avec des pyréthrinoïdes, notament la deltaméthrine (résistance croisée).

Les mécanismes impliqués dans cette résistance n’ont été mis en évidence que dans le village Boutoto (dans le département du Kouilou) en 2009. Des études faites dans d'autres pays ont montré que la résistance croisée au DDT et aux pyréthrinoïdes était due à l'implication du gène de résistance kdr et des enzymes métaboliques (Djègbè et al., 2011; Gnanguenon et al., 2014; Konaté et al., 2016).

L’étude de Boutoto en 2009 a montré que la résistance au DDT était due aux mutations L1014F et L1014S. Tandis que la résistance aux pyréthrinoïdes était principalement attribuée aux monooxygénases et aux estérases. (Koekemoer et al., 2011).

Concernant le bendiocarbe, les tests faits à Boutoto en 2009 ont montré une baisse de sensibilité à cet insecticide. D'autres tests devront être faits pour confirmer le statut de résistance à cet insecticide. A Brazzaville en 2013, toutes les populations testées ont été sensibles au bendiocarbe tandis qu'en 2018, les tests faits à Brazzaville avec des individus issus des sites de maraichage et à Djoumouna ont montré des niveaux de résistance élevés. Le bendiocarbe n'étant pas ou peu utilisé au Congo en santé publique, l'apparition de cette résistance suppose une utilisation des carbamates et des organophosphorés en agriculture dans ces localités. En effet, à Djoumouna, l'une des principales activités est le maraichage, l'emploi et l'effet des insecticides ne sont donc pas à exclure. La résistance au bendiocarbe a aussi été mise en évidence au Cameroun dans des sites de maraichage et des sites pollués (Antonio-Nkondjio 
et al., 2016). Cette résistance a été attribuée à une exposition fréquente aux xénobiotiques, y compris aux insecticides appartenant aux familles des carbamates et des organophosphorés. En revanche, toutes les populations de moustiques testées ont été sensibles au malathion. Cette sensibilité serait due à la non utilisation de cet insecticide en santé publique et en agriculture.

La résistance au bendiocarbe et au pirimiphos-méthyl est préoccupante car ces insecticides peuvent être utilisés en pulvérisation intradomiciliaire (PID) dans les localités où la résistance aux pyréthrinoïdes a été mise en évidence dans le cadre de la gestion de la résistance. En effet au Bénin, le bendiocarbe a montré son efficacité quand il a été utilisé dans les PID (Akogbéto et al., 2010).

La présente étude rapporte pour la première fois la résistance au bendiocarbe et au pirimiphos-méthyl au Congo. Ce constat fait apparaitre la nécessité d’identifier les mécanismes de résistance impliqués. Ce qui permettra d'adopter des mesures appropriées de gestion de la résistance afin de réduire le risque d'échecs potentiels des interventions de lutte antivectorielle basées sur l'emploi des pyréthrinoïdes.

Les tests avec les synergistes ont été réalisés sur des populations résistantes à la deltaméthrine et au DDT. La préexposition au PBO et au TPP a restauré la sensibilité d'An. gambiae s.l. à la deltaméthrine. La mortalité à la deltaméthrine a été significativement améliorée en utilisant le TPP impliquant les estérases dans la résistance aux pyréthrinoïdes. Par contre, aucune différence des taux de mortalité n’a été observée en exposant ces populations au diethylmaleate, ce qui montre que les glutathion-S-transférases ne sont pas impliquées dans la résistance aux pyréthrinoïdes. Les individus résistants au DDT ont tous survécu après leur exposition au PBO, au maléate de diéthyle et au TPP ; cela suggère que les enzymes detoxifiantes ne sont pas impliquées dans la résistance au DDT. L'augmentation des taux de mortalité a été liée à l'action des synergistes PBO et TPP respectivement inhibiteurs des monooxygénases et d'estérase. Ce qui suggère une implication importante des monooxygénases et des estérases dans la résistance des anophèles testés aux pyréthrinoïdes (deltaméthrine) en favorisant la pénétration des insecticides dans le corps du moustique et en neutralisant la métabolisation de l'insecticide (OMS, 2017). Ces résultats sont en accord avec ceux obtenus par d'autres auteurs qui ont montré que l'effet combiné des synergistes et des pyréthrinoïdes améliore la sensibilité des vecteurs aux pyréthrinoïdes (Chouaïbou et al., 2014; Gleave et al., 2017; Fagbohun et al., 2019). Ces résultats montrent que l'utilisation des MIILDA avec du PBO serait une alternative efficace contre les vecteurs résistants aux pyréthrinoïdes dans le cadre de la gestion de la résistance à ces insecticides. 


\section{Conclusion}

Cette étude montre la répartition de la résistance d'An. gambiae s.l. aux insecticides utilisés en santé publique au Congo et révèle les variations spatiales et temporelles des profils de résistance aux insecticides. Cette résistance pourrait trouver ses origines dans l'utilisation des insecticides depuis la période coloniale (principalement le DDT) en santé publique, en agriculture et celle à large échelle des MIILDA depuis une trentaine d'années au Congo.

Un plaidoyer pour une coordination entre le ministère de la santé (programme de lutte antivectorielle) et les autres départements concernés par l'utilisation des insecticides (agriculture, environnement, commerce, etc.) est nécessaire pour une mise en œuvre efficiente des stratégies de gestion de la résistance. En outre, ces résultats montrent que la surveillance n’est pas régulière. Ainsi pour corriger cette faiblesse, la gestion de cette résistance obligerait une surveillance accrue et régulière, et la caractérisation des mécanismes sous-jacents de celle-ci. Pour garantir l'efficacité des MIILDA dans la lutte contre le paludisme au Congo, il serait souhaitable de préserver les pyréthrinoïdes pour l'imprégnation des moustiquaires et réduire leur utilisation dans les autres domaines.

\section{Remerciements}

Les auteurs présentent leur vif remerciement au Docteur Jean Mermoz YOUNDOUKA, Coordonnateur du Programme National de Lutte contre le Paludisme au Ministère de la Santé et de la Population, pour la mise à disposition des données récapitulatives dudit programme, portant sur la sensibilité d'Anopheles gambiae s.l. aux insecticides.

\section{References:}

1. Abbott, W.S. (1925) A method of computing the effectiveness of an insecticide. J econ Entomol 18: 265-267.

2. Abuelmaali, S.A., Elaagip, A.H., Basheer, M.A., Frah, E.A., Ahmed, F.T., Elhaj, H.F., et al. (2013) Impacts of agricultural practices on insecticide resistance in the malaria vector Anopheles arabiensis in Khartoum State, Sudan. PLoS One 8: e80549.

3. Adam, J.-P. (1964) Répartition géographique des anophèles en République du Congo (Brazzaville). Cah ORSTOM Sér Ent Méd Parasitol 2: 73-82.

4. Akogbéto, M. and Yakoubou, S. (1999) Résistance des vecteurs du paludisme vis-à-vis des pyréthrinoïdes utilisés pour l'imprégnation des moustiquaires au Bénin, Afrique de l'Ouest. Bull Soc Pathol Exot 92: 123-30. 
5. Akogbéto, M.C., Padonou, G.G., Gbénou, D., Irish, S., and Yadouleton, A. (2010) Bendiocarb, a potential alternative against pyrethroid resistant Anopheles gambiae in Benin, West Africa. Malaria journal 9: 204.

6. Alou, L.P.A., Koffi, A.A., Adja, M.A., Tia, E., Kouassi, P.K., Koné, M., and Chandre, F. (2010) Distribution of ace-1 R and resistance to carbamates and organophosphates in Anopheles gambiae ss populations from Côte d'Ivoire. Malaria Journal 9: 167.

7. Antonio-Nkondjio, C., Fossog, B.T., Kopya, E., Poumachu, Y., Djantio, B.M., Ndo, C., et al. (2015) Rapid evolution of pyrethroid resistance prevalence in Anopheles gambiae populations from the cities of Douala and Yaoundé (Cameroon). Malaria journal 14: 155.

8. Antonio-Nkondjio, C., Fossog, B.T., Ndo, C., Djantio, B.M., Togouet, S.Z., Awono-Ambene, P., et al. (2011) Anopheles gambiae distribution and insecticide resistance in the cities of Douala and Yaoundé (Cameroon): influence of urban agriculture and pollution. Malaria Journal 10: 154.

9. Antonio-Nkondjio, C., Poupardin, R., Tene, B.F., Kopya, E., Costantini, C., Awono-Ambene, P., and Wondji, C.S. (2016) Investigation of mechanisms of bendiocarb resistance in Anopheles gambiae populations from the city of Yaoundé, Cameroon. Malaria journal 15: 424.

10. Bitsindou, P. (2014) Assessment of the susceptibility of malaria vectors to insecticides in some localities of Congo (Brazzaville, Kinkala, Owando and Ewo), Brazzaville, Congo: Ministère de la santé et de la population.

11. Bitsindou, P., Bagayoko, M., and Manga, L. (2006) Profil entomologique du paludisme au Congo.

12. Brogdon, W.G. and Barber, A.M. (1990) Microplate assay of glutathione S-transferase activity for resistance detection in singlemosquito triturates. Comparative biochemistry and physiology B, Comparative biochemistry 96: 339-342.

13. Chandre, F., Darrier, F., Manga, L., Akogbeto, M., Faye, O., Mouchet, J., and Guillet, P. (1999) Situation de la résistance aux pyréthrinoïdes chez Anopheles gambiae sensu lato.

14. Chareonviriyaphap, T., Rongnoparut, P., Chantarumporn, P., and Bangs, M.J. (2003) Biochemical detection of pyrethroid resistance mechanisms in Anopheles minimus in Thailand. Journal of vector ecology: journal of the Society for Vector Ecology 28: 108-116.

15. Chouaïbou, M., Zivanovic, G.B., Knox, T.B., Jamet, H.P., and Bonfoh, B. (2014) Synergist bioassays: A simple method for initial metabolic 
resistance investigation of field Anopheles gambiae sl populations. Acta tropica 130: 108-111.

16. Cisse, M.B., Keita, C., Dicko, A., Dengela, D., Coleman, J., Lucas, B., et al. (2015) Characterizing the insecticide resistance of Anopheles gambiae in Mali. Malaria journal 14: 327.

17. CNEV (2014) Utilisation des insecticides et gestion de la résistance.

18. Djègbè, I., Boussari, O., Sidick, A., Martin, T., Ranson, H., Chandre, F., et al. (2011) Dynamics of insecticide resistance in malaria vectors in Benin: first evidence of the presence of L1014S kdr mutation in Anopheles gambiae from West Africa. Malaria journal 10: 261.

19. Fagbohun, I.K., Oyeniyi, T.A., Idowu, T.E., Otubanjo, O.A., and Awolola, S.T. (2019) Cytochrome P450 Mono-Oxygenase and Resistance Phenotype in DDT and Deltamethrin-Resistant Anopheles gambiae (Diptera: Culicidae) and Culex quinquefasciatus in Kosofe, Lagos, Nigeria. Journal of medical entomology 56: 817-821.

20. Gillies, M., T. and De Meillon, B. (1968) The Anophelinae of Africa south of the Sahara (Ethiopian zoogeographical region). The South African Institue for Medical Research,. johannesburg.

21. Gillies, M.T. and Coetzee, M. (1987) A supplement to the Anophelinae of Africa South of the Sahara, The South African Institue for Medical Research. Johannesburg.

22. Gleave, K., Lissenden, N., Richardson, M., and Ranson, H. (2017) Piperonyl butoxide (PBO) combined with pyrethroids in long-lasting insecticidal nets (LLINs) to prevent malaria in Africa. Cochrane Database of Systematic Reviews.

23. Gnanguenon, V., Govoetchan, R., Agossa, F.R., Ossè, R., Oke-Agbo, F., Azondekon, R., et al. (2014) Transmission patterns of Plasmodium falciparum by Anopheles gambiae in Benin. Malaria journal 13: 444.

24. Itoua, Motandi, Bitsindou, P., and Zoulani, A. (2003) Evaluation de la sensibilité d'Anopheles gambiae s.l. à trois pyréthrinoïdes et un organochloré dans sept chefs lieux départementaux du CONGO 2002 - 2003, Brazzaville, Congo: Ministère de la santé et de la population.

25. Jones, C.M., Toé, H.K., Sanou, A., Namountougou, M., Hughes, A., Diabaté, A., et al. (2012) Additional selection for insecticide resistance in urban malaria vectors: DDT resistance in Anopheles arabiensis from Bobo-Dioulasso, Burkina Faso. PloS one 7: e45995.

26. Koekemoer, L.L., Spillings, B.L., Christian, R.N., Lo, T.-C.M., Kaiser, M.L., Norton, R.A., et al. (2011) Multiple insecticide resistance in anopheles gambiae (Diptera: Culicidae) from pointe Noire, Republic of the Congo. Vector-Borne and Zoonotic Diseases 11: 1193-1200. 
27. Konan, K.G., Koné, A.B., Konan, Y.L., Fofana, D., Konan, K.L., Diallo, A., et al. (2011) Résistance d'Anopheles gambiae sl aux pyréthrinoïdes et au DDT à Tiassalékro, village de riziculture irriguée en zone sud forestière de Côte-d'Ivoire. Bulletin de la Société de pathologie exotique 104: 303.

28. Konaté, L., Diallo, M., Faye, O., and Dia, I. (2016) Patterns of insecticide resistance and knock down resistance $(\mathrm{kdr})$ in malaria vectors An. arabiensis, An. coluzzii and An. gambiae from sympatric areas in Senegal. Parasites \& vectors 9: 71.

29. Kucuker, H., Sahin, O., Yavuz, Y., and Yürümez, Y. (2009) Fatal Acute Endosulfan Toxicity: A Case Report. Basic \& Clinical Pharmacology \& Toxicology 104: 49-51.

30. MEFDDE (2017) Circulaire $\mathrm{N}^{\circ} 0614 / \mathrm{MEFDDE} / \mathrm{CAB} / \mathrm{DGE}$ : Précisant les conditions de délivrance des autorisations relatives aux importations aux exportations, à la formulation, au commerce et à l'utilisation des produits chimiques à usage industriel.

31. Ndo, C., Menze-Djantio, B., and Antonio-Nkondjio, C. (2011) Awareness, attitudes and prevention of malaria in the cities of Douala and Yaoundé (Cameroon). Parasites \& vectors 4: 181.

32. OMS (2014) Guide entomologique du paludisme, Geneva, Switzerland.

33. OMS (2017) Procédures pour tester la résistance aux insecticides chez les moustiques vecteurs du paludisme - seconde édition, Geneva, Switzerland.

34. PNLP (2018a) Directives nationales de prévention et de prise en charge du paludisme.

35. PNLP (2016) Rapport synthèse évaluation de la sensibilité des vecteurs du paludisme aux insecticides, Brazzaville, Congo: Ministère de la santé et de la population.

36. PNLP (2018b) Rapport d'activités 2017, Brazzaville, Congo: Ministère de la santé et de la population.

37. PNLP (2010) Rapport insecticide, Brazzaville, Congo: Ministère de la santé et de la population.

38. PNLP (2012) Rapport Technique De la Campagne de Distribution Universelle de la Moustiquaire Imprégnée d'insecticide, Brazzaville, Congo: Ministère de la santé et de la population.

39. Thiaw, O., Doucouré, S., Sougoufara, S., Bouganali, C., Konaté, L., Diagne, N., et al. (2018) Investigating insecticide resistance and knock-down resistance (kdr) mutation in Dielmo, Senegal, an area under long lasting insecticidal-treated nets universal coverage for 10 years. Malaria journal 17: 123. 
40. WHO (1975) Manual on practical entomology in malaria. Part 2, Methods and techniques, Geneva, Switzerland.

41. WHO (1998) Test procedures for insecticide resistance monitoring in malaria vectors, bio-efficacy and persistence of insecticides on treated surfaces, Geneva, Switzerland.

42. WHO (2019) World malaria report 2019, Geneva, Switzerland.

43. William, G.B. and Janet, C. (1997) Heme peroxidase activity measured in single mosquitoes identifies individuals expressing an elevated oxidase for insecticide resistance. Journal of the American Mosquito Control Association 13: 233-237.

44. Yahouédo, G.A., Cornelie, S., Djègbè, I., Ahlonsou, J., Aboubakar, S., Soares, C., et al. (2016) Dynamics of pyrethroid resistance in malaria vectors in southern Benin following a large scale implementation of vector control interventions. Parasites \& vectors 9: 385. 